\title{
Impresión 3D para la elaboración de arrecifes artificiales. Registro térmico másico
}

\section{D printing for the production of artificial reefs. Thermal mass registration}

Presentación: 06 y 07/10/2020

\section{Doctorando:}

\section{Adrian I. Yoris-Nobile}

Grupo de Investigación en Tecnología de los Materiales de Construcción y Calidad (GINTEMAC) Universidad Tecnológica Nacional Argentina / Grupo de Investigación de Tecnología de la Construcción (GITECO) Universidad de Cantabria - España

ayoris@frsf.utn.edu.ar - yorisai@unican.es

\section{Director/es:}

\section{María Josefina Positieri - Elena Blanco Fernandez}

\section{Co-director/es:}

\section{Bárbara Belén Raggiotti}

\begin{abstract}
Resumen
En el presente trabajo, correspondiente a la tesis doctoral en curso, se muestra la elaboración de arrecifes artificiales mediante una impresora 3D tipo Delta y el uso de estructuras auxiliares, como pallets, cercos perimetrales y arena de relleno, para lograr imprimir las formas irregulares. Como material de impresión se utilizaron dos tipos de morteros de cemento, uno con arena caliza y otro con arena de vidrio triturado. En dichos morteros se empleó cemento de bajo contenido en Clinker, se incorporó fly ash como adición y se empleó árido reciclado, lo cual dotó a los moteros de sustentabilidad o bajo impacto ambiental. En los arrecifes artificiales se registró la temperatura interna y de borde para conocer su evolución durante un período entorno a los 5 días. Estas determinaciones se realizaron en dos piezas de la misma forma y con distintos morteros, además se evaluó una pieza prismática maciza. Los resultados muestran que la metodología empleada para la elaboración de piezas irregulares fue adecuada, ya que el modelo digital de los arrecifes artificiales se pudo reproducir en forma fidedigna y no se registró asentamiento del mortero durante el proceso. En cuento a las temperaturas registradas, se observó un aumento de temperatura superior a los $50^{\circ} \mathrm{C}$ en el interior, y superior a $40^{\circ} \mathrm{C}$ en el borde, este comportamiento fue muy similar en ambos tipos de morteros y un poco superior en la pieza de control.
\end{abstract}

Palabras clave: Impresión 3D, arrecifes artificiales, registro térmico.

\begin{abstract}
This paper, corresponding to the doctoral thesis in progress, shows the elaboration of artificial reefs using a 3D Delta-type printer and the use of auxiliary structures, such as pallets, perimeter fences and filler sand, to achieve the printing of irregular shapes. Two types of cement mortar were used as printing material, one with limestone sand and the other with crushed glass sand. In these mortars, low clinker content cement was used, fly ash was incorporated as an addition and recycled aggregate was used, which provided the bikers with sustainability or low environmental impact. In the artificial reefs, internal and border temperatures were recorded to know their evolution during a period of about 5 days. These determinations were made in two pieces of the same shape and with different mortars, in addition a solid prismatic piece was evaluated. The results show that the methodology used for the elaboration of irregular pieces was adequate, since the digital model of the artificial reefs could be reproduced in a reliable way and no settlement of the mortar was registered during the process. Regarding the recorded temperatures, an increase of more than $50^{\circ} \mathrm{C}$ was observed in the interior, and more than $40^{\circ} \mathrm{C}$ in the border. This behavior was very similar in both types of mortar and a little higher in the control piece.
\end{abstract}


Keywords: 3D printing, artificial reefs, thermal registration.

\section{Introducción}

El empleo de impresión 3D en el ámbito de la construcción, como las tecnologías basadas en Extruded Material Systems (EMS) presentan una gran versatilidad, por lo que permitirían la realización de estructuras con diseños geométricos más complejos, optimizando formas, ejecutando construcciones sin el uso de encofrados, reduciendo el desperdicio de materiales y optimizando recursos (El-Sayegh et al, 2020; Khoshnevis \& Epstein, 2004).

En este sentido, el Artificial Reef 3D Printing for Atlantic Area (3DPARE) es un proyecto europeo que tiene como objetivo principal el diseño y fabricación de arrecifes artificiales mediante impresión 3D, para ser sumergidos posteriormente en las costas del Atlántico Norte. Las técnicas de impresión 3D se consideran las más adecuadas para la elaboración de arrecifes artificiales, ya que permitirían realizar diseños complejos, de gran importancia para la habitabilidad de la fauna marina y de la colonización biológica (Cast Ege et al, 2016). En línea con el proyecto, se conoce que el sector de la construcción es una de las fuentes principales de contaminación ambiental, generando grandes cantidades de desecho y emitiendo grandes cantidades de dióxido de carbono a la atmósfera (Lehne \& Preston, 2018). Por este motivo, el empleo de cementos con bajo contenido de Clinker, el uso de adiciones provenientes de desechos industriales y la incorporación de áridos reciclados, son acciones que permiten lograr sostenibilidad en la industria. (Medina et al, 2016; Sadiqul Islam et al, 2017).

En este trabajo se describe la metodología empleada para lograr imprimir piezas en 3D de formas irregulares con huecos en su interior, correspondientes a arrecifes artificiales, mediante el empleo de morteros sostenibles. Durante el proceso de impresión de los arrecifes, se registraron las temperaturas internas y de borde de dichas piezas.

\section{Desarrollo}

Para la elaboración de los arrecifes artificiales se emplearon dos tipos de morteros de cemento, uno con arena caliza (CL), de granulometría 0-3 mm y otro con reemplazo de la arena caliza por arena de vidrio triturada (CG), de granulometría 0-0.3 mm. En la Tabla 1 se muestran las dosificaciones de los morteros empleados, en los cuales, además, se utilizó cemento tipo III/B $32.5 \mathrm{~N}$-SR, el cual es un cemento de alto horno, con un contenido de escoria siderúrgica entre un $66 \%$ y $80 \%$, según noma, aunque lo habitual es que ronde el $66 \%$, lo que se traduce en un contenido de Clinker muy bajo, entorno al 31\%, con resistencia media $32.5 \mathrm{MPa}$ a 28 días, resistencia inicial normal [N] y resistente a sulfatos [SR] (UNE-EN_197-1, 2011). Se emplearon caolín y fly ash como adición; este último es un subproducto industrial de las centrales térmicas, producto de la quema del carbón, con disponibilidad a nivel mundial en grandes cantidades (Xie \& Ozbakkaloglu, 2015). Y como aditivo se empleó superplastificante (S.P.).

Tabla 1. Dosificación de los morteros empleados

\begin{tabular}{lrr}
\hline & \multicolumn{1}{c}{ CL } & \multicolumn{1}{c}{ CG } \\
{$\left[\mathrm{kg} / \mathrm{m}^{3}\right]$} & {$\left[\mathrm{kg} / \mathrm{m}^{3}\right]$} \\
\hline Cem III/B & 525.39 & 507.01 \\
Caliza [0-3] & 1050.78 & 507.01 \\
Vidrio [0-0.3] & - & 507.01 \\
Agua & 280.47 & 289.08 \\
S.P. & 4.38 & 3.97 \\
Fly ash & 262.69 & 253.50 \\
Caolín & 21.89 & 21.13 \\
\hline
\end{tabular}

Para la fabricación de los arrecifes artificiales se empleó una impresora 3D tipo Delta basada en la tecnología EMS. Dicho equipo cuenta con una tolva de carga, en la cual se vierte el mortero a extrudir o imprimir y mediante un tornillo sin fin, ubicado en su interior, se transfiere el material a la boquilla de impresión, cuya salida tiene una sección circular de $20 \mathrm{~mm}$ de diámetro. 
La metodología que se estableció para la fabricación de los arrecifes artificiales, consistió inicialmente en la colocación de un pallet reforzado como base, para que una vez impresa la pieza, se pudiese retirar la misma mediante una carretilla hidráulica. Una vez ubicado el pallet, se colocó un cerco de madera perimetral y se comenzó la impresión. A medida que se avanzaba capa a capa con la impresión, se fue colocando arena de sílice de granulometría 1-2 mm, la cual actuaba como soporte y relleno de la estructura impresa. Este proceso descrito se repitió en forma consecutiva a medida que se avanzaba con la impresión, colocándose nuevos cercos hasta completar la altura del arrecife. En la Figura 1 se muestran las secuencias de avance en la fabricación de los mismos.
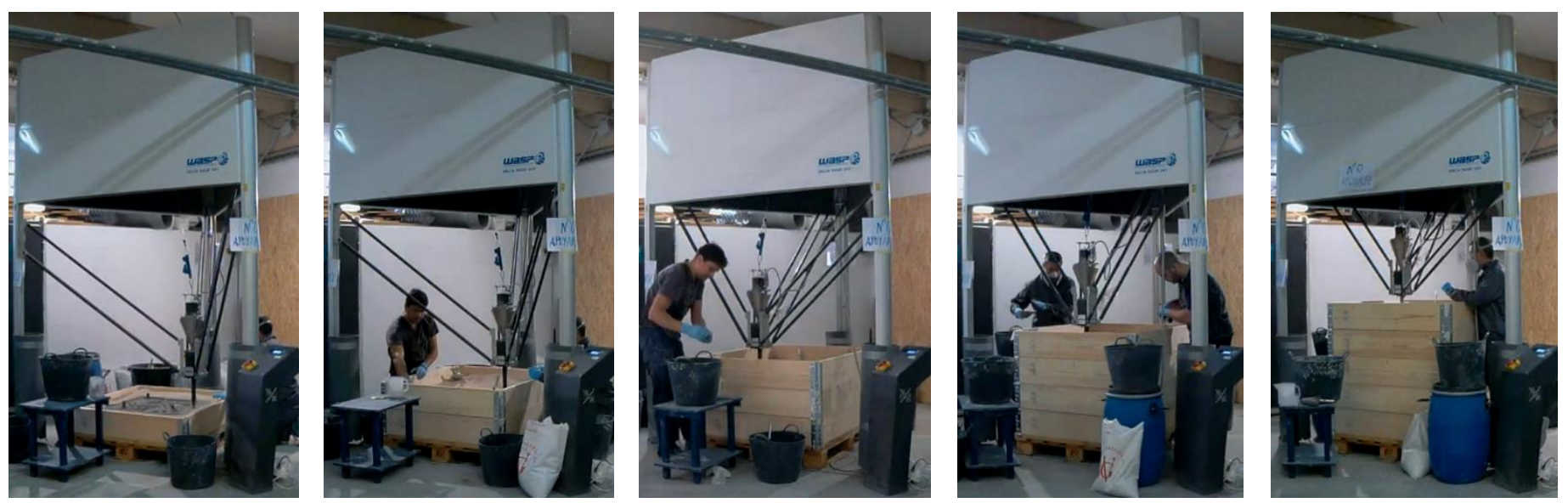

Figura 1. Metodología para la elaboración de los arrecifes artificiales. Secuencia de avance en la fabricación.

La elección de esta metodología de trabajo, se justifica por el hecho de que las piezas a imprimir tenían protuberancias en su perímetro exterior, así como huecos en su interior, con el objetivo de atraer una mayor biodiversidad al momento de ser sumergidos, lo cual generaba que las capas de impresión que se iban depositando en forma sucesiva, no coincidieran una sobre la otra; esto provocaba inestabilidad en el mortero y el colapso de la estructura. Por este motivo se empleó arena de sílice como material de soporte y relleno provisional, para que en aquellos lugares donde la pieza tuviese paredes con ángulos inclinados o incluso bóvedas internas, tuviera una base en donde se apoyaran las capas sucesivas. Por su parte, los cercos de madera perimetrales se emplearon para contender la arena de relleno y servir de embalaje definitivo para su transporte a destino.

El proyecto consistió en la elaboración de 36 arrecifes artificiales que tenían distintas formas y diferentes terminaciones superficiales, cuyos tamaños estaban inscriptos en un área de impresión de $95 \mathrm{~cm}$ de diámetro, una altura de $90 \mathrm{~cm}$ y un peso promedio de cada pieza de $850 \mathrm{~kg}$. En este trabajo se analizaron los arrecifes artificiales con la forma denominada Cubic Big (CB) y Cubic Control (CC), elaborados con los morteros CL y CG los primeros (arrecifes CB) y con mortero CL los segundos (arrecifes CC). La forma CB se muestra en la

Figura 2, donde se observan, además, los cortes de sección en coincidencia con los huecos de cada nivel. Por su parte, la forma CC corresponde a una pieza prismática maciza de $77 \mathrm{~cm}$ de lado por $85 \mathrm{~cm}$ de altura.
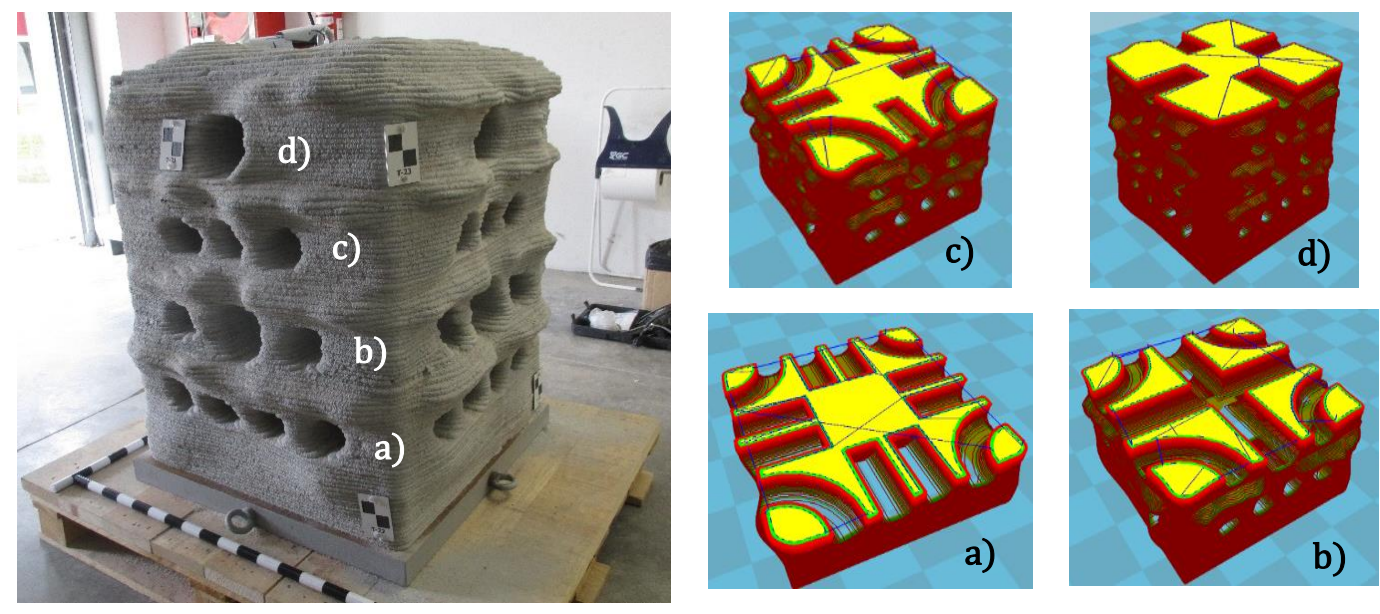

Figura 2. Arrecife con la forma Cubic Big (CB) y cortes de secciones en coincidencia con los huecos de cada nivel. 
Para recoger los datos de temperaturas, se empleó un equipo formado principalmente por una placa de desarrollo con microcontrolador AVR y un módulo lector de tarjeta microSD para el almacenamiento de datos (Figura 3a). Para registrar dichos datos de temperatura se utilizaron sondas de temperatura DS18B20 con un rango de funcionamiento de $-55^{\circ} \mathrm{C}$ a $+125^{\circ} \mathrm{C}$, con una precisión de $\pm 0.5^{\circ} \mathrm{C}$ en el rango de $-10^{\circ} \mathrm{C}$ a $+85^{\circ} \mathrm{C}$. La ubicación transversal de las sondas en los arrecifes artificiales se muestra en la Figura 3b), en donde se dispusieron dos sondas por cada punto a ser registrado. En tanto que la ubicación vertical de estas sondas, coincide en la zona media entre los huecos del nivel c) y d). El equipo empleado permitía, además, registrar la humedad relativa y temperatura ambiente. La toma de datos se realizó con una frecuencia de un minuto por un período de tiempo de 5 días.
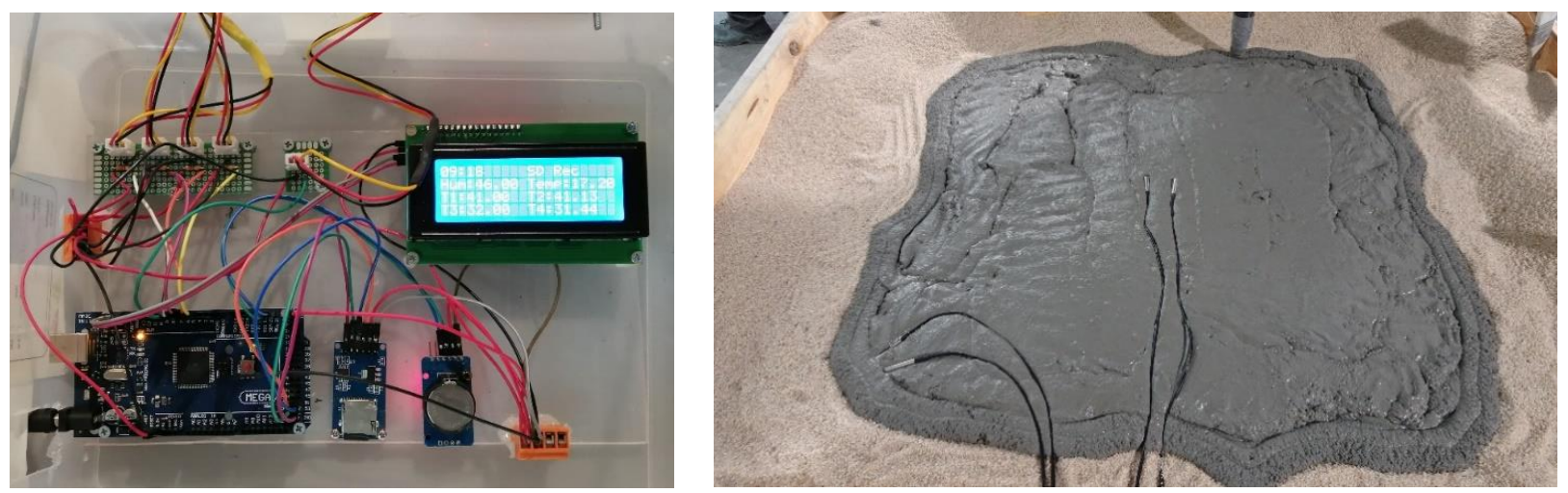

Figura 3. a) Equipo para el registro de temperaturas y humedad. b) Ubicación de las sondas de temperatura en el arrice CB.

\section{Resultados}

Los resultados muestran que las temperaturas en el interior (T.I.) de los arrecifes artificiales se encuentran entre los $50^{\circ} \mathrm{C}$ y $55^{\circ} \mathrm{C}$, en tanto que las temperaturas en los bordes (T.B.) se encuentran entre los $40^{\circ} \mathrm{C}$ y $45^{\circ} \mathrm{C}$ (Figura 4, Figura 5, Figura 6). Por su parte, los valores de temperatura ambiente (T.amb.) se mantienen en torno a los $20^{\circ} \mathrm{C}$ y la humedad relativa (H.R.) oscila entre $50 \%$ y $60 \%$. Las curvas de temperatura registradas, muestran un marcado ascenso hasta el entorno de las $30 \mathrm{~h}$ y luego comienza un lento descenso que tiende a estabilizarse llegando a las 120 h. Los picos de temperatura máxima T.I. y T.B. se encuentran desplazados en el tiempo, por lo que, para determinar el gradiente térmico máximo, se toman los registros en el momento en el que se detecta el máximo valor de T.I.

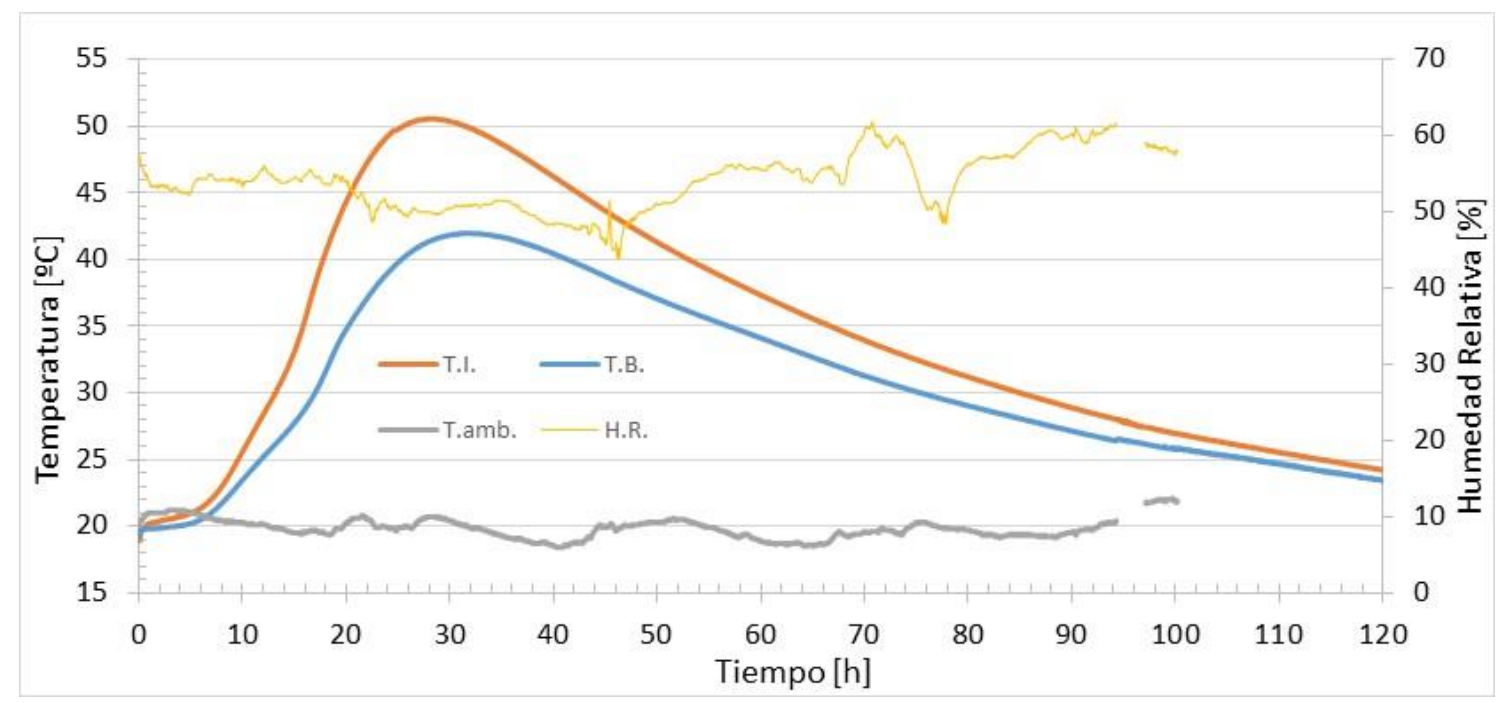

Figura 4. Registro de temperaturas y humedad relativa en el arrecife artificial CB-CL.

En el caso particular de los arrecifes artificiales CB-CL se registró una T.I. máxima de 50.6ำ y una T.B máxima de $42^{\circ}$ C, obteniéndose un gradiente térmico máximo de 9.3 C (Figura 4). En el caso de los arrecifes artificiales CB-CG se registró una T.I. máxima de $50.2^{\circ} \mathrm{C}$ y una T.B máxima de $39.5^{\circ} \mathrm{C}$, obteniéndose un gradiente térmico máximo de 
11.5ำ C (Figura 5). Por último, en el arrecife de control CC-CL se registró una T.I. máxima de 52.1ํ C y una T.B máxima de $43.7^{\circ} \mathrm{C}$, obteniéndose un gradiente térmico máximo de 9.4ํㅡ $\mathrm{C}$ (Figura 6).

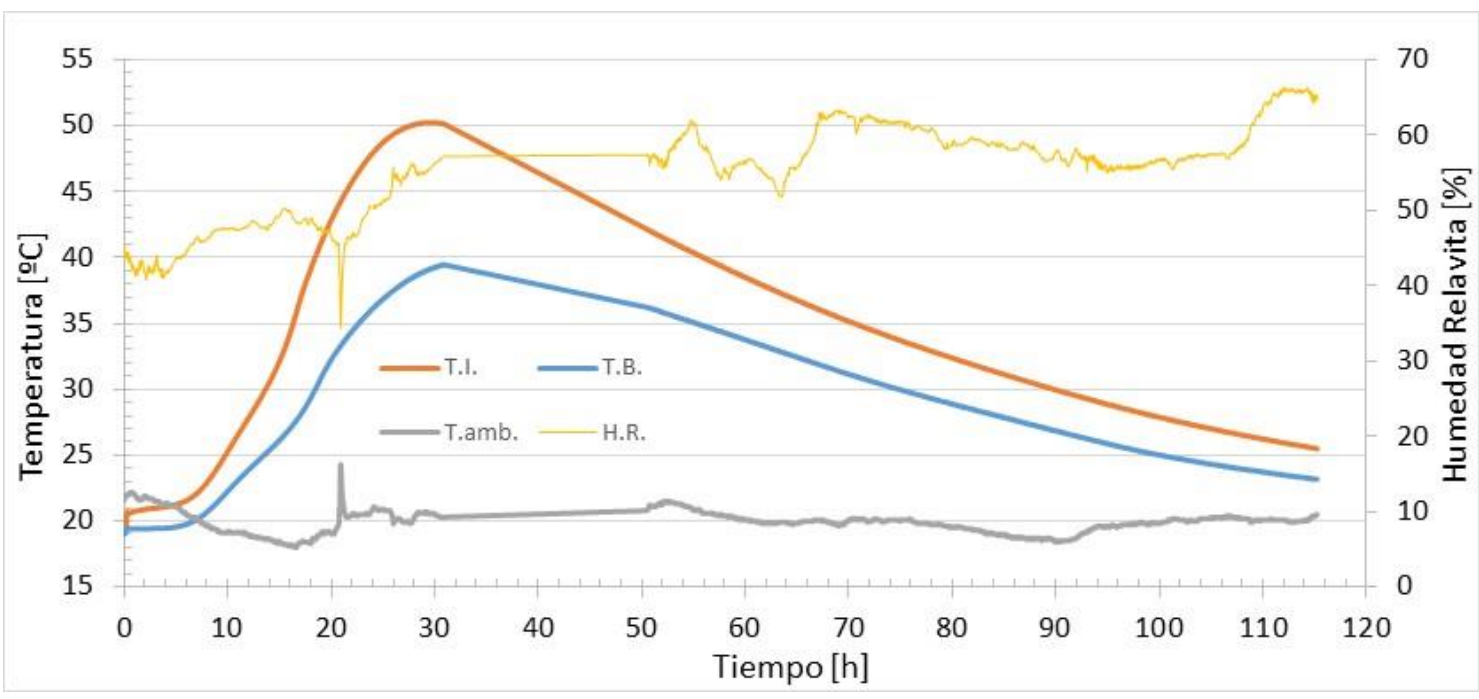

Figura 5. Registro de temperaturas y humedad relativa en el arrecife artificial CB-CG.

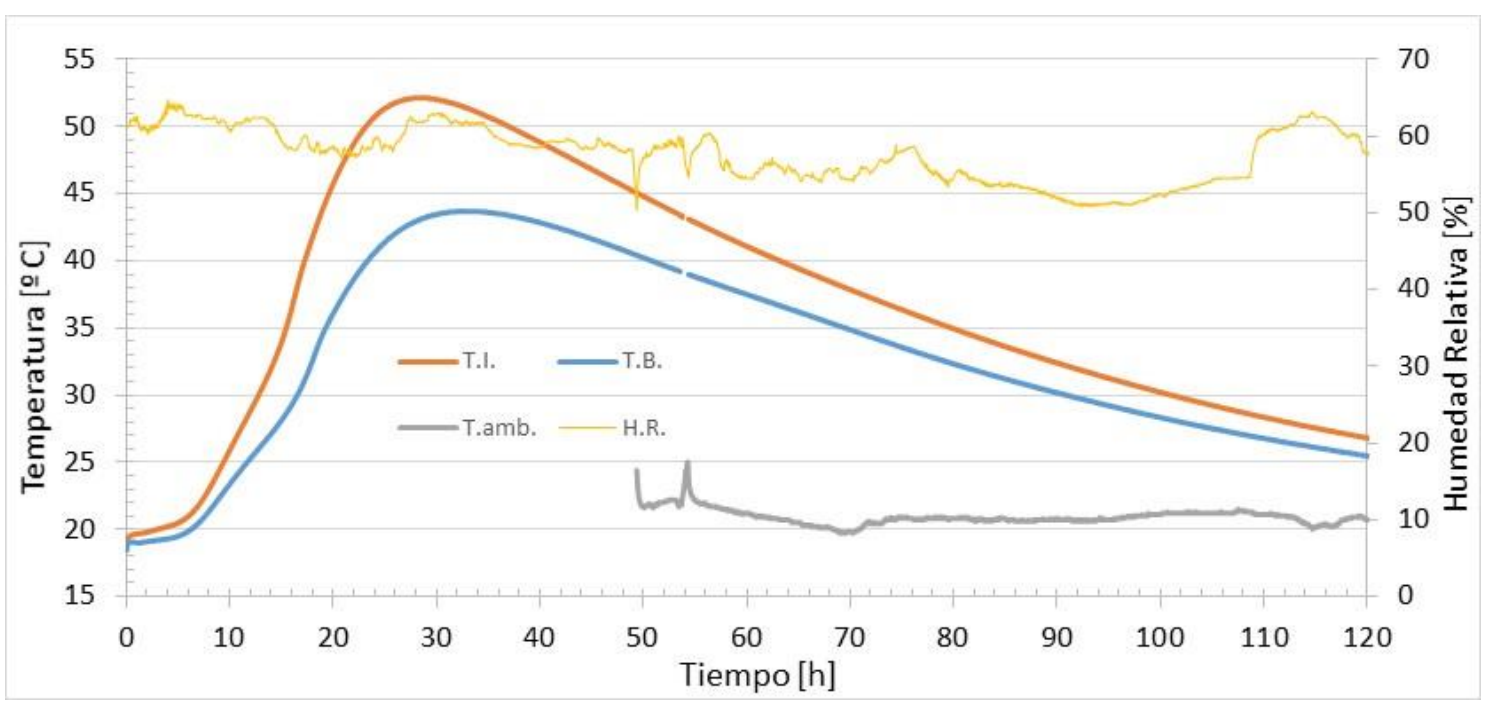

Figura 6. Registro de temperaturas y humedad relativa en el arrecife artificial CC-CL.

De los resultados anteriores, se observa que no hay una diferencia térmica significativa en las curvas de temperatura correspondientes a los distintos morteros empleados, CL y CG. Sin embargo, se observa un aumento en el gradiente térmico en los morteros CG con respecto a los morteros $\mathrm{CL}, 11.5^{\circ} \mathrm{C}$ y $9.3^{\circ} \mathrm{C}$ respectivamente.

La evolución de las curvas de temperatura en los arrecifes tiene un comportamiento similar a las obtenidas en los hormigones masivos cuando se realizan controles de temperatura con termocuplas. Haciendo una analogía con estos hormigones, la (ACI_207.1R., 2005) define al hormigón masivo como "cualquier volumen de hormigón con dimensiones lo suficientemente grande como para requerir que se tomen medidas para atender la generación de calor proveniente de la hidratación del cemento y los cambios de volumen para minimizar la fisuración". Traducido a la práctica, se entiende como hormigón masivo a una estructura cuyo lado de menor dimensión sea mayor a $70 \mathrm{~cm}$. En nuestro caso, el arrecife CC se encuentra en el límite de estas dimensiones, por lo que podría caer dentro de la clasificación de estructura masiva. Pero por otro lado, la (ACI_201.2R, 2016) indica que, cuando en las estructuras no se registren temperaturas mayores a $70^{\circ} \mathrm{C}$, no es necesario tomar acciones, ya que no existe riego de fisuración o microfisuración por tensión térmica o dilatación térmica diferencial. En los arrecifes artificiales, las temperaturas registradas no superan los 55을 $\mathrm{C}$, por lo que no existe riesgo de daño por estos fenómenos. 
Cabe destacar aquí, que los registros de temperatura en los arrecifes artificiales se realizaron con la arena de sílice que cubría completamente a los arrecifes. Este hecho, pudo provocar que las temperaturas registradas alcancen un mayor valor, al igual que puedo haber evitado una mayor diferencia de gradiente térmica entre el interior y borde de los arrecifes, debido a que pudo colaborar en que la disipación de calor de las piezas impresas sea menor.

Otra consideración a realizar, corresponde al tipo de cemento empleado. Según la norma (UNE-EN_14216, 2015), el Cem III/B, por su composición, podría considerarse un cemento de bajo calor de hidratación, sin embargo, la clasificación comercial no incluye la designación de bajo calor de hidratación (LH). De todos modos, más allá del bajo contenido de Clinker del cemento, entorno al 31\%, la cantidad de cemento por metro cúbico es muy elevada, superando los $500 \mathrm{~kg} / \mathrm{m}^{3}$ y sumado a que las escorias de alto horno pueden tener propiedades aglomerantes per se, son las razones que podrían justificar las altas temperaturas registradas en los arrecifes artificiales impresos.

Finalmente, se puede concluir que la metodología empleada para la elaboración de piezas irregulares fue adecuada, ya que se logró reproducir fielmente el modelo digital de los arrecifes artificiales. En cuanto a las temperaturas desarrolladas en los arrecifes, no representaron un problema estructural, pero sí es un parámetro a tener en cuenta en caso de elaborar piezas de mayores dimensiones.

\section{Agradecimientos}

Este Proyecto ha sido co-financiado por el European Regional Development Fund a través del Interreg Atlantic Area Programme, en el marco del proyecto 3DPARE (EAPA_174/2016). Este trabajo refleja la opinión de los autores, por lo que las autoridades del programa no se hacen responsables del uso de la información aquí incluida. Los autores quieren agradecer también a las siguientes empresas: Solvay, por el suministro de las cenizas volantes; BASF, por el suministro de los aditivos utilizados en la investigación; FCC ámbito, por el suministro de vidrio reciclado triturado y al Grupo Cementos Portland Valderribas (planta Mataporquera) por el suministro del cemento.

\section{Referencias}

ACI_201.2R. Guide to Durable Concrete (2016). American Concrete Institute.

ACI_207.1R. Mass Concrete (2005). ACI Committee report. Retrieved from http://dl.mycivil.ir/dozanani/ACI/ACI 207.1R-05 Guide to Mass Concrete_MyCivil.ir.pdf

Cast Ege, I., Milon, E., Erard Fourneau, G., \& Tauzia, A. (2016). First results of fauna community structure and dynamics on two artificial reefs in the south of the Bay of Biscay (France). Estuarine, Coastal and Shelf Science, 179, 172-180. https://doi.org/10.1016/j.ecss.2016.02.015

El-Sayegh, S., Romdhane, L., \& Manjikian, S. (2020, June 1). A critical review of 3D printing in construction: benefits, challenges, and risks. Archives of Civil and Mechanical Engineering. Springer. https://doi.org/10.1007/s43452020-00038-w

Khoshnevis, B., \& Epstein, D. J. (2004). Automated construction by contour crafting-related robotics and information technologies. Automation in Construction, 13(1), 5-19. https://doi.org/10.1016/j.autcon.2003.08.012

Lehne, J., \& Preston, F. (2018). Concrete Change Innovation in Low-carbon Cement and Concrete (Chatham Ho). London: The Royal Institute of International Affairs. Retrieved from https://reader.chathamhouse.org/makingconcrete-change-innovation-low-carbon-cement-and-concrete\#

Medina, C., Sáez del Bosque, I. F., Asensio, E., Frías, M., \& Sánchez de Rojas, M. I. (2016). New additions for ecoefficient cement design. Impact on calorimetric behaviour and comparison of test methods. Materials and Structures, 49(11), 4595-4607. https://doi.org/10.1617/s11527-016-0809-5

Sadiqul Islam, G. M., Rahman, M. H., \& Kazi, N. (2017). Waste glass powder as partial replacement of cement for sustainable concrete practice. International Journal of Sustainable Built Environment, 6(1), 37-44. https://doi.org/10.1016/j.ijsbe.2016.10.005

UNE-EN_14216. Cemento - Composición, especificaciones y criterios de conformidad de los cementos especiales de muy bajo calor de hidratación (2015). AENOR - Asociación Española de Normalización y Certificación.

UNE-EN_197-1. Cemento. Parte 1: Composición, especificaciones y criterios de conformidad de los cementos comunes. (2011). AENOR - Asociación Española de Normalización y Certificación.

Xie, T., \& Ozbakkaloglu, T. (2015). Behavior of low-calcium fly and bottom ash-based geopolymer concrete cured at ambient temperature. Ceramics International, 41(4), 5945-5958.

https://doi.org/10.1016/j.ceramint.2015.01.031 